\title{
TRANSFORMATION OF PROBABILITIES
}

\author{
JULIAN H. BLAU ${ }^{1}$
}

1. Transformation. Let $R$ be a $\sigma$-algebra ${ }^{2}$ of subsets of $X$, and $\beta$ the set of all probability measures $P$ on $R$. Let $T$ transform $P$ into itself. For certain sets $E \in R$, knowledge of $P$ throughout $E$ (i.e., for all subsets of $E$ belonging to $R$ ) determines $T P$ throughout $E$. The class of sets having this property will be denoted by $E_{T}$, or better, since $T$ will be fixed, by $E$. Evidently $E$ contains $0, X$, and the complements of atoms. We show that if $E$ is sufficiently large, then $T$ is a linear combination of the identity and a constant. There are applications to the theory of learning and to political theory $[1 ; 3 ; 4 ; 6]$.

THEOREM 1. (A) If $E$ contains an algebra $\boldsymbol{A}$ whose Borel extension is $R$, and if $|R|>4$, then $T P \equiv \alpha P+(1-\alpha) P_{0}$, where $\alpha \leqq 1$ and $P_{0} \in P$.

(B) The converse is true with no restriction on $R$.

(C) If $R$ is infinite, then $\alpha \geqq 0$.

In the political interpretation, the elements of $X$ are parties (or political positions). $P$ is the distribution of voters, $T$ is the electoral mechanism, and $T P$ the distribution of seats in the legislature. If $T$ is the identity, the mechanism is Proportional Representation. If $T$ is a constant, the political complexion of the legislature is fixed by law. It will be seen from Theorem 3 that $E \in E$ means that if the complement $-E$ unites in a coalition, the effect is independent of whether this occurs before or after the election. $|R|>4$ means essentially that there are more than two parties. Part (A) of the theorem is not true for $|R|=4$.

In learning theory, $P$ is a probability distribution of responses, and $T P$ is a new distribution resulting from a learning experience. If $T$ is the identity, there is no learning. If $T$ is a constant, this is one-trial learning.

Bush, Mosteller, and Thompson [4] proved an equivalent theorem for the case $R$ finite and $E=R$ (Corollary 3 of Theorem 3). Some of their ideas are used in the proof.

Denote by $B$ the class of sets $E$ such that $P(E)=Q(E)$ implies $T P(E)=T Q(E)$, for all $P, Q \in \mathcal{P}$. The importance of $B$ is that for

Presented to the Society April 8, 1961; received by the editors August 22, 1960.

1 Supported by the National Science Foundation.

${ }^{2}$ Borel field. We follow the terminology of [5]. In addition, the Borel extension of a class $\boldsymbol{M}$ of sets is the smallest $\sigma$-algebra containing $\boldsymbol{M}$. This is the same as $\boldsymbol{S}(\boldsymbol{M})$ if $X$ is the countable union of sets in $M$. 
each proper set $E \in B$, there is a function $\gamma_{E}$ mapping $[0,1]$ into itself such that $T P(E)=\gamma_{E}[P(E)]$. We have also $\gamma_{0}(0)=0$.

For any class $S$ of subsets of $X$, let $S^{*}$ denote the class of sets $E$ for which the complement $-E \in S$.

Proposition 1. $E \cap E^{*} \subseteq B$.

Proof. Let $E,-E \in E$. Let $P(E)=Q(E)$ for two members $P, Q$ of P. Define $P^{\prime} \in \mathcal{P}$ as follows. For $A \in R$, let $P^{\prime}(A)=P(A-E)$ $+A(c) P(E)$, where $c \in E$ and $A(x)$ is the characteristic function of $A$. Define $Q^{\prime}$ similarly. We have $P^{\prime} \equiv Q^{\prime}$ on $E$. Also $P \equiv P^{\prime}$ and $Q \equiv Q^{\prime}$ on $-E$. Hence $T P^{\prime} \equiv T Q^{\prime}$ on $E$, while $T P \equiv T P^{\prime}$ and $T Q \equiv T Q^{\prime}$ on $-E$. In particular, the last two equations are true for $-E$ itself, and, taking complements, also for $E$. We have $T P(E)=T P^{\prime}(E)=T Q^{\prime}(E)$ $=T Q(E)$, proving $E \in B$.

Since $A$ is an algebra, $A=A^{*}$. Thus $B \supseteq E \cap E^{*} \supseteq A \cap A^{*}=A$, and so $B \supseteq A$.

Define the set function $u$ on the class $A-\{X\}$ as follows: $u(E)$ $=\gamma_{E}(0)$. Using the fact that $T P$ is a measure for each $P$, and choosing $P$ so as to vanish on the appropriate sets, it is easy to show that $u$ is a measure on the semiring $\boldsymbol{A}-\{X\}$, and therefore extends uniquely to a measure $u$ on $\boldsymbol{R}[\mathbf{5} ; \mathbf{7}]$. Evidently $u \leqq 1$ on $\boldsymbol{A}-\{X\}$, but it would be incorrect to infer that $u(X) \leqq 1$.

Proposition 2. Let $E \cap F=0 ; E, F, E \cup F$ proper sets in $B ; x, y$, $x+y \in[0,1]$. Then $\gamma_{E \cup F}(x+y)=\gamma_{E}(x)+\gamma_{F}(y)$.

Proof. Using all the hypotheses, it is easy to show that there is a probability measure $P$ with $P(E)=x$ and $P(F)=y$. For this $P$,

$$
\gamma_{E \cup F}(x+y)=T P(E \cup F)=T P(E)+T P(F)=\gamma_{E}(x)+\gamma_{F}(y) .
$$

If $E$ and $F$ are proper sets in $A$, let $E \sim F$ denote the statement that $\gamma_{E}(x)-u(E) \equiv \gamma_{F}(x)-u(F)$ for all $x \in[0,1] . \sim$ is an equivalence relation.

PROPOSITION 3. The relation $\sim$ is universal on the proper sets in $\boldsymbol{A}$.

Proof. (1) Let $E \subset B$, where $\subset$ denotes proper inclusion. By Proposition 2, with $F=B-E$ and $y=0, \gamma_{B}(x)=\gamma_{E}(x)+\gamma_{B-E}(0)$. Letting $x=0, \gamma_{B}(0)=\gamma_{E}(0)+\gamma_{B-E}(0)$. Subtracting, we have $E \sim B$.

(2) If $E \cap F=0$ and $E \cup F \neq X$, then $E \sim E \cup F \sim F$ by (1).

(3) If $E, F$ are incomparable and $E \cap F \neq 0$, then $E \sim E \cap F \sim F$ by (1).

(4) This leaves only the case $F=-E$. For the first time, we invoke the hypothesis $|R|>4$, which easily implies $|A|>4$. Hence $E$ or $F$ 
must have a proper subset, say $E \supset A$. Then $E \sim A$ by (1) and $A \sim F$ by (2).

In view of Proposition 3 and $|\boldsymbol{A}|>2$, the equation

$$
\gamma(x)=\gamma_{E}(x)-u(E) \quad \text { for } E \in A-\{0, X\}
$$

defines $\gamma(x)$ uniquely. $\gamma$ maps $[0,1]$ into $[-1,1]$.

Proposition 4. $\gamma(x) \equiv \alpha x$, with $\alpha \leqq 1$.

Proof. Let $x, y, x+y \in[0,1]$. Choose $E, F$ so that $E, F, E \cup F$ are proper sets in $A$, and so that $E \cap F=0$. Here we have used $|\boldsymbol{R}|>4$ for the second and last time. By Proposition 2 and the definitions of $u$ and $\gamma$,

$$
\begin{aligned}
\gamma(x+y)+u(E \cup F) & =\gamma_{E \cup F}(x+y)=\gamma_{E}(x)+\gamma_{F}(y) \\
& =\gamma(x)+u(E)+\gamma(y)+u(F) .
\end{aligned}
$$

Since $u$ is additive, we conclude that $\gamma(x+y)=\gamma(x)+\gamma(y)$. A bounded function of this type is of the stated form. The proof in [2] can be adapted. Obviously, $\alpha \leqq 1$.

Thus $T P(E)=\alpha P(E)+u(E)$ for all $E$ in the semiring $\boldsymbol{A}-\{X\}$. If $\alpha \geqq 0$, then $\alpha P+u$ is a measure on $R$, equal to $T P$ on $A-\{X\}$, and therefore on $R$. If $\alpha \leqq 0$, then $T P-\alpha P$ is a measure on $R$, equal to $u$ on $\boldsymbol{A}-\{X\}$, and therefore on $\boldsymbol{R}$. In either case $T P=\alpha P+u$ on $\boldsymbol{R}$. In passing, note that

$$
1=T P(X)=\alpha+u(X) .
$$

If $\alpha=1$, then $T P \equiv P$. If $\alpha<1$, define $P_{0}$ by $(1-\alpha) P_{0}=u$. The main assertion (A) of Theorem 1 follows.

Assertion (B) is immediate, taking $\boldsymbol{A}=\boldsymbol{R}$. For (C) we require a simple result from set theory. We omit the proof, which is not difficult.

LemмA. If $\boldsymbol{A}$ is an infinite algebra of subsets of $X$, then $X$ is the union of a monotone sequence of sets of $A-\{X\}$.

To resume the proof of $(C)$, the infinite cardinality of $R$ implies the same for $\boldsymbol{A}$. Then we have $u(X)=\lim _{n \rightarrow \infty} u\left(E_{n}\right)$ for sets $E_{n} \in A$ $-\{X\}$. But $u \leqq 1$ on $A-\{X\}$, and therefore $u(X) \leqq 1$. With (1), we have $\alpha \geqq 0$.

For applications to special cases, we need the following closure properties of $E$, which are of independent interest.

Theorem 2. (A) If $E, F \in E$, and $E \cup F \neq X$, then $E \cap F \in E$.

(B) $E$ is closed with respect to countable union. 
Proof. (A) Let $P \equiv Q$ on $E \cap F$. Without loss of generality, assume $P(E) \leqq Q(E)$. Define a new probability measure $P^{\prime}$ as equal to $P$ on $E$ (i.e., throughout $E$ ), equal to $Q$ on $F-E$, and arbitrary on $X-E-F$ except that $P^{\prime}(E)+P^{\prime}(F-E)+P^{\prime}(X-E-F)=1$. For other sets, $P^{\prime}$ is defined by additivity. In verification that the values assigned on $X-E-F$ are feasible, we observe that this set is not empty and that $P^{\prime}(E \cup F) \leqq Q(E \cup F) \leqq 1$.

Now $P \equiv P^{\prime}$ on $E$ and $Q \equiv P^{\prime}$ on $F$. Hence $T P \equiv T P^{\prime}$ on $E$ and $T Q \equiv T P^{\prime}$ on $F$. The last two identities are true, therefore, on $E \cap F$. Hence $T P \equiv T Q$ on $E \cap F$, and $E \cap F \in E$.

We remark that when $E \cup F=X$, (A) is false in the strong sense that given such overlapping incomparable $E, F$, there exists a $T$ for which $E$ and $F$ are in $E_{T}$, but $E \cap F$ is not.

(B) Let $P \equiv Q$ on $E=\bigcup_{1}^{\infty} E_{n}$, where $E_{n} \in E$. Then $P \equiv Q$ on $E_{n}$, which implies $T P \equiv T Q$ on $E_{n}$, for each $n$. Let $\left\{F_{n}\right\}$ be a disjoint sequence having the same partial unions as $\left\{E_{n}\right\}$. We have $T P \equiv T Q$ on $F_{n}$, since $F_{n} \subseteq E_{n}$. Then $T P \equiv T Q$ on $E$ by countable additivity, and $E \in E$.

The hypothesis of Theorem 1 may be expressed in two parts:

(I) $|\boldsymbol{R}|>4, E$ contains a class $\boldsymbol{S}$ whose Borel extension is $\boldsymbol{R}$, and $X \in S$.

(II) $S$ is a ring.

Proposition 5. In Theorem 1, (II) can be weakened to: $\mathbf{S}$ is a semiring.

Proof. The class of finite disjoint unions of elements of $S$ is a ring [5]. Since it contains $S$, this ring generates $R$ and contains $X$. By Theorem 2B, $E$ contains the ring.

Examples. In all of these, let $R$ be the class of Borel sets.

(i) $X=$ the real line. Let $E$ contain all intervals $[\alpha, \beta)$. (Here and in the following it would suffice to take $\alpha$ and $\beta$ rational.) Then $E$ contains also $[\alpha, \infty)$ and $(-\infty, \alpha)$. With 0 and $X$, these finite and semi-infinite intervals constitute a semiring. Proposition 5 applies, and $T P \equiv \alpha P+(1-\alpha) P_{0}$ with $0 \leqq \alpha \leqq 1$ as in Theorem 1 . This is equally true if $E$ is assumed instead to contain all proper closed intervals.

(ii) (a) $X=(0,1)$, (b) $X=[0,1]$, (c) $X=[0,1)$. Similar to Example (i).

(iii) $X=$ Euclidean $n$-space $(n>1)$. Let $E$ contain all half spaces $\left\{x: x_{i} \geqq \alpha\right\}$ and $\left\{x: x_{i}<\alpha\right\}$. Then $E$ contains all finite intersections of these sets. (This implication is false for $n=1$.) With $X$ added, these constitute a semiring, Proposition 5 applies, and $T$ has the form 
stated in Theorem 1. This is true also if $E$ is assumed to contain all slices $\left\{x: x_{i} \in[\alpha, \beta)\right\}$, or alternatively all cells

$$
\left\{x: x_{i} \in\left[\alpha_{i}, \beta_{i}\right], i=1, \cdots, n\right\} .
$$

2. Combination. Bush and Mosteller [3] raised the question in learning theory of whether a set $E$ could be shrunk to a point without making $T$ ambiguous on the reduced space. More precisely, let $E \in R$. A transformation $C$ of $\beta$ into itself is called a combination of $E$ if it satisfies

$$
C P \equiv P \text { on }-E
$$

and

$$
P(E)=Q(E) \text { implies } C P \equiv C Q \text { on } E \text {. }
$$

For example, let $c \in E$, and let

$$
C P(A)=P(E-A)+A(c) P(E) \quad \text { for each } A \in \boldsymbol{R} .
$$

We say that $E \in C$ ( $E$ is combinable) if for each combination $C$ of $E$, and for each $P \in \mathcal{P}$, we have

$$
C T C P=C T P .
$$

In learning theory, (3) is called the Combining of Classes condition.

Theorem 3. $\mathrm{C}=E^{*}$.

Proof. Let $E \in C$, and $C$ be a combination of $E$. We observe first that (C1) and (C2) imply

$$
C P=C Q \text { if and only if } P \equiv Q \text { on }-E \text {. }
$$

Now let $P \equiv Q$ on $-E$. Then $C P=C Q$. Hence $C T P=C T C P=C T C Q$ $=C T Q$. Then a second use of (C3) yields $T P \equiv T Q$ on $-E$. This proves $C \subseteq E^{*}$.

Let $-E \in E$. Let $C$ combine $E$. Then $P \equiv C P$ on $-E$, and therefore $T P \equiv T C P$ on $-E$. By (C3), $C T P=C T C P$. Thus $E^{*} \subseteq C$.

COROLlARY 1. In Theorem 1, the hypothesis that $E$ contains the algebra $\boldsymbol{A}$ can be replaced by $\boldsymbol{C} \supseteq \boldsymbol{A}$.

Corollary 2. (A) The union of two overlapping sets of $\mathbf{C}$ is in $\mathbf{C}$.

(B) $\mathrm{C}$ is closed with respect to countable intersection.

COROllary 3. Let $X$ be finite, $|X|>2$, let $R$ be the class of all subsets of $X$, and $\mathbf{C}=\boldsymbol{R}$. Then $T P \equiv \alpha P+(1-\alpha) P_{0}$.

This is the Bush-Mosteller-Thompson theorem [4] mentioned 
earlier. Bush and Mosteller [3] showed that $\alpha(|X|-1) \geqq-1$. This bound is attained.

Regarding Example (ii)(c) as the real numbers modulo 1, let $\mathbf{C}$ contain all intervals $[\alpha, \beta)$, naturally including the case $\alpha<1<\beta$. With 0 , this class is a semiring $S$. Since $S=S^{*}$, also $E \supseteq S$, so that Proposition 5 applies, and $T$ has the familiar form of Theorem 1. In Example (i) (the real line) the corresponding implication is false, even with the additional assumption that $\boldsymbol{C}$ contains all semi-infinite intervals, and similarly for Example (ii). To prove this, we use

Proposition 6. Let $T$ be a combination of $E$ of type (2). Then

$$
\boldsymbol{C}_{T}=[\{\{c\}\}+\cup\{E\}-\cup\{-E\}-] \cap \boldsymbol{R} .
$$

(For any subset $S$ of $X,\{S\}-$ and $\{S\}+$ denote respectively the class of subsets of $S$ and the class of supersets of $S$.) The proof is omitted.

Returning to Example (i), let $T$ be that combination of $E=[c, \infty$ ) of type (2) which concentrates $P(E)$ at $c$. We see that $\boldsymbol{C}$ contains all the finite and infinite intervals mentioned above, but that intervals $[\alpha, \beta)$ containing $c$ are not in $E$, and the conclusion of Theorem 1 is false here.

3. Partition. A related problem, motivated by learning theory and political theory, is the following. For $n>1$, let $\otimes_{n}$ denote the class of all partitions of $X$ into exactly $n$ nonempty parts $X_{i}$, and let $\mathfrak{e}_{n}$ denote the subclass of partitions (called combinable) for which the $n$-tuple $\left[P\left(X_{1}\right), \cdots, P\left(X_{n}\right)\right]$ uniquely determines $\left[T P\left(X_{1}\right), \cdots, T P\left(X_{n}\right)\right]$. It is not difficult to show that if each $X_{i} \in C$, then $\left(X_{1}, \cdots, X_{n}\right) \in \mathbb{e}_{n}$. The converse is false, so that the latter statement is actually weaker than the former. Despite this, we have

TheOREM 4. If $\mathfrak{C}_{n}=\mathcal{P}_{n}$ for some $n<\log _{2}|\boldsymbol{R}|$, then $\boldsymbol{E}=\boldsymbol{C}=\boldsymbol{R}$, and $T P \equiv \alpha P+(1-\alpha) P_{0}$.

Proof. First we show that $E \in E$ for all $E$ divisible into $n-1$ (proper) parts. We can assume $E \neq X$. Let $P \equiv Q$ on $E$, and let $A \subseteq E$. We can express $A$ as $\bigcup_{1}^{a} A_{i}$, where either $a=n-1$ or each $A_{i}$ is atomic. If $a<n-1$, then $E-A=\bigcup_{a+1}^{n-1} A_{i}$ by the hypothesis on $E$. In either case, $P\left(A_{i}\right)=Q\left(A_{i}\right)$ for $i=1, \cdots, n-1$ and $P(-E)=Q(-E)$. Hence $T P\left(A_{i}\right)=T Q\left(A_{i}\right)$. Summing from 1 to $a, T P(A)=T Q(A)$, and $E \in E$.

Evidently $E \in E$ is proved unless $E$ consists of the union of fewer than $n-1$ atoms. Let $E$ be the union of $n-2$ atoms. Since $|R|>2^{n}$, $-E$ has three parts, $A, B, C$. Then $E \cup A$ and $E \cup B$ are in $E$, their 
union is not $X$, and so their intersection $E$ is in $E$ by Theorem $2 \mathrm{~A}$. Similarly for $n-3$, etc. Thus $E=R$, and the remaining statements follow from Theorems 1 and 3.

The theorem is false for $n \geqq \log _{2}|R|$.

Let $g_{n}$ denote the class of partitions of $X$ into $n$ nonempty parts $X_{i}$, each of which is in a fixed semiring $\mathbf{S}$ whose Borel extension is $\boldsymbol{R}$. (The notation is suggested by examples where $\boldsymbol{S}$ consists of intervals.)

When $X$ is the real line, and $S$ the class of intervals $[\alpha, \beta),(-\infty, \alpha)$, $[\alpha, \infty)$, the example at the end of $\S 2$ shows that $0 \neq g_{n} \subseteq \mathcal{C}_{n}$ for all $n$ does not imply $E=R$. The same is true for $X$ a finite interval. The situation is different for a circle.

THEOREM 5. Let $X$ be the set of real numbers modulo 1 , and $\mathbf{S}$ the class of intervals $[\alpha, \beta)$. If $\mathfrak{g}_{n} \subseteq \mathfrak{e}_{n}$ for some $n$, then $E=C=R$, and $T P$ $\equiv \alpha P+(1-\alpha) P_{0}$.

Proof. If $n=2$, then evidently $S \subseteq B$. With the single exception of Proposition 3, the proof of Theorem 1A applies, with the semiring $\boldsymbol{S}$ replacing the algebra $\boldsymbol{A}$. Proposition 1 is superfluous. We show now that the conclusion of Proposition 3 holds also in the present context. All intervals mentioned are proper, i.e., not 0 or $X$.

(1) Let $I_{1} \subset I$. If $I-I_{1}$ is an interval, then $I_{1} \sim I$ as in Proposition 3. If $I-I_{1}$ is not an interval, then it is the union of two disjoint intervals $I_{2}$ and $I_{3}$. Moreover, $I_{1} \cup I_{2}$ is an interval. Thus $I_{1} \sim I_{1} \cup I_{2} \sim I$.

(2) If $I_{1} \cap I_{2}=0$ and $I_{1} \cup I_{2} \neq X$, then there is a proper interval $I$ containing $I_{1} \cup I_{2}$. Then $I_{1} \sim I \sim I_{2}$ by (1).

Thus (1) and (2) in the proof of Proposition 3 are true in our present case. (3) and (4) apply unchanged. (This proof that $S \subseteq B$ implies the linearity of $T$ is valid also for $X=$ the real line with $S$ all $[\alpha, \beta)$, and for $X=$ Euclidean $n$-space with $S$ all semiclosed cells.) This completes the proof for $n=2$.

Next, let $n>2$. Note that $P \equiv Q$ on $I$ if and only if $P \equiv Q$ for all subintervals of $I$ touching an end point. Hence $I \in E$ if and only if the equality of $P$ and $Q$ for all such subintervals implies the same for $T P$ and $T Q$.

Let $P \equiv Q$ on $I$, and let $I_{1}$ be a subinterval touching an end point. Write the interval $I-I_{1}$ as the disjoint union $\bigcup_{3}^{n} I_{j}$, and let $I_{2}=-I_{1}$. (Here we have used the fact that $\mathbf{S}=\boldsymbol{S}^{*}$.) We have $\left(I_{1}, \cdots, I_{n}\right) \in \mathfrak{e}_{n}$, and $P\left(I_{j}\right)=Q\left(I_{j}\right)$ for all $j$. Hence $T P\left(I_{j}\right)=T Q\left(I_{j}\right)$ for all $j$, and in particular for $j=1$. Since $I_{1}$ was arbitrary, this proves $I \in E$. Thus $S \subseteq E$.

Using $S=S^{*}$ again, we have $S \subseteq E \cap E^{*}$. By Proposition $1, S \subseteq B$, and the first part of the proof applies. 


\section{REFERENCES}

1. J. H. Blau, The combining of classes condition in learning theory, Technical Report No. 32, Applied Mathematics and Statistics Laboratories, Stanford University, 1960.

2. R. P. Boas, A primer of real functions, New York, Wiley, 1960.

3. R. R. Bush and F. Mosteller, Stochastic models for learning, New York, Wiley, 1955.

4. R. R. Bush, F. Mosteller and G. L. Thompson, A formal structure for multiplechoice situations, Chapter 8 in R. M. Thrall, C. H. Coombs and R. L. Davis (editors), Decision processes, New York, Wiley, 1954.

5. P. R. Halmos, Measure theory, New York, Van Nostrand, 1950.

6. R. D. Luce and H. Raiffa, Games and decisions, New York, Wiley, 1957.

7. J. von Neumann, Functional operators. Vol. I. Measures and integrals, Princeton, Princeton University Press, 1950.

Antioch College 